\title{
Advantages of Interactive Visualization Tools in Planning Tasks
}

\author{
A.A. Zakharova ${ }^{1}$, E.V. Vekhter ${ }^{2}$, A.V. Shklyar ${ }^{2}$ \\ zaa@tu-bryansk.ru|vehter@tpu.ru|shklyarav@tpu.ru \\ ${ }^{1}$ Bryansk State Technical University, Bryansk, Russian Federation; \\ ${ }^{2}$ Tomsk Polytechnic University, Tomsk, Russian Federation
}

The paper proposes the use of visualization tools as an independent or complementary tool designed to solve problems related to the planning and audit of the results of various processes. The advantages arising as a result of transition to attraction of visual perception for formation of the General idea of process and its results existing in the form of heterogeneous data are shown. The use of visualization tools to find contradictions and errors made at the stage of process design is proposed. The proposed tool for visualizing an educational environment is supplemented by an ability to save options for solving planning problems and for corresponding real results. This creates conditions for a planning search for periods of varying lengths, during which the search and evaluation of factors that have necessary effect on the achieved results of the educational program are carried out.

Keywords: visual analytics, visual model, data analysis, visual interpretation, visual perception.

\section{Introduction}

The basis of the "solving the planning problem" definition in current article is an idea of the sequence of actions taken to achieve the predetermined goal. The goal is the state of the studied system, characterized by the set of values which are characteristics of the system. Thus, the solution to the planning problem is the description of the process, which ends with obtaining the state of the $\{\mathrm{Pi}\}$ system with accuracy satisfying the decision maker (DM).

While solving problems of the indicated type, or when it becomes necessary to compare the achieved results and the resources expended, the task is to jointly analyze data included in individual elements descriptions of the investigated (planned) process. Data analysis aims to achieve two goals:

1. Identification of contradictions between disparate information descriptions of processes included in the main process;

2. Determination of possible ways and selection of the best ones, in terms of resource intensity, to improve the achieved results.

The initial data in the analysis is the set of various data that characterize the studied process - heterogeneous data. Interpretation of such data is aimed at revealing implicit patterns in heterogeneous descriptions of processes and at obtaining new knowledge. Systematization of efforts made by a researcher in carrying out the analysis of heterogeneous data, aimed at formalizing procedures for setting and solving similar problems, it will make it possible to generalize the accumulated experience and simplify the interpretation of the initial data. An element of the educational program - curriculum for bachelors was taken as the initial data in this article (Fig. 1).

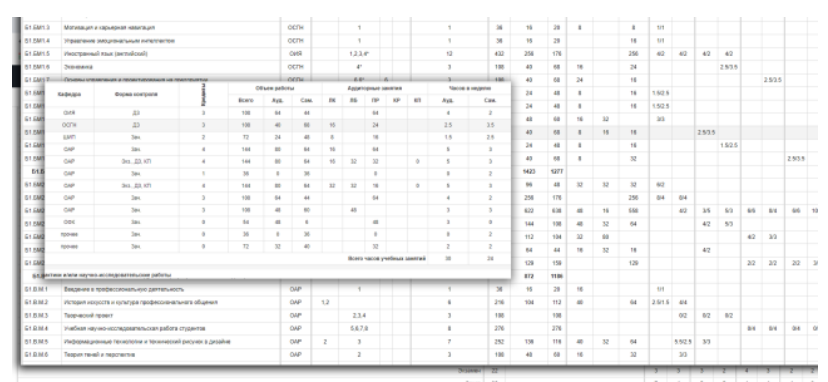

Fig. 1 Example of educational planning data

Large amount of input data and limitation of available resources (computational, time, human) often become a problem of practical analysis of heterogeneous data while designing and controlling results of various processes. Traditional means of interpretation and analysis have several disadvantages [2, 4]:
1. High requirements for specialist's qualifications involved in the analysis, which increase overall resource intensity for searching the necessary solution.

2. The need in interdisciplinary interaction between researchers complicates the organization of analysis and increases the risk of interpretation errors.

3. While solving applied problems associated with the audit of achieved results, there is a need to search and eliminate internal contradictions in the studied data, which source is errors made earlier at the planning stage. Subjective nature of such contradictions complicates their detection by traditional means of analysis based on previously formulated criteria.

\section{Formal statement of the problem}

The analyzed process $\mathrm{E}$ is an ordered set of elements $O=\left\{O_{1 . .} O_{n}\right\}$, where $O_{i}$ is an elementary process defined by a set of unique properties. Properties of the element $\mathrm{O}_{\mathrm{i}}$ are divided into two subsets, based on their functional differences:

$$
O_{i}=\left\{P_{i}\right\}=\left\{\operatorname{In}_{x}, \text { Out }_{y}\right\},
$$

where the subset $\left\{I_{x}\right\}$ is process requirements, incoming connections of $O_{i}$ element, and the subset $\left\{O_{u t}\right\}$ is results of the process, outgoing connections of $O_{i}$ element. Connections of the $O_{i}$ element determined in this way are its main characteristics, which ensure interaction with other elements and achievement of the goal of the main process $E$.

In accordance with these definitions, the goal of the main process $E$ can be represented as a composition of outgoing connections, characterized by some parameters, for example, the number of outgoing connections (results) and some weighting factors. Thus, the output of the set $O=\left\{O_{1 . .} O_{n}\right\}$ is determined by the predetermined goal $G=\{O u t\}$ and available resources.

The formal goal of solving the planning problem formulated in this way is to find the set $O$ that provides given or best results while observing all boundary requirements of $B$ : $O=\left\{O_{n}: n=\min , G=\max \right\}$

In the simplest case, when properties of elements $O_{i}$ are specified, the goal of the solution is to order the set $O$, which ensures the fulfillment of the requirement (1). In the opposite case, the solution to the problem is a new set $O^{*}=\left\{I n *, O u t^{*}\right\}$ which corresponds to requirements of $B$.

\section{Data Features}

The amount of data and a number of additional information have an influence on the interpretation process of the studied data. Some information is not directly related to the problem solving $[3,5]$. At the stage of preliminary analysis of the initial data or during the process of solving the problem the analyst gets a lot of additional information (intermediate solutions, verification data, classification and clustering results, etc.). In 
addition, the process of researching and interpreting of data, which requires significant time resources (hours, days), is associated with the need to study new data - replacing or complementing the old ones.

History of data acquisition, methods of their collection, the presence of errors, partial lack of data, etc. could have a significant impact on the research objective achievement. Metadata - second-ordered data, play the role of an additional source of information for the researcher and are a part of heterogeneous data. Thus, the peculiarity of studying heterogeneous data which are into the user's disposal while solving a planning problem is the need to compare many heterogeneous descriptions of individual elements of the process, taking into account intermediate versions of solutions and the need to update interpreted data during analysis $[1,8]$. As a perspective way to overcome these difficulties, the use of data visualization tools with the ability of organizing interactive interaction between a user and the initial data is proposed.

\section{User's abilities}

The need to use perceptual capabilities, characterized by empirical principles, sets to a developer a task of determination of the set of user characteristics that are purposefully involved in the interpretation of the visual image of data. Selection can be based on a preliminary assessment of available resources, which include:

1. Characteristics of a potential user, whose involvement in the process of data interpretation does not create prerequisites for increasing its duration.

2. Computing resources, which allow to obtain visual images of data, if they meet the requirement of interactivity with them.

3. Temporary resources that determine speed of construction and interpretation of visual data images.

4. Additional requirements arising from the statement of the research task, including: prior knowledge of a user, his qualifications, probable features of perception, etc.

The set of user's characteristics involved in the cognitive interpretation of the visual image of the studied data can be determined by generalization existing schemes of the visualization process [9]. Based on the obtained set, three groups of visualization tools are distinguished, which differ in functional purpose and in methods of practical implementation:

1. Observation. Obtaining visual information (perception of color, space, movement, allocation of groups, recognition of forms, signs).

2. Search. That means to identify relevant objects and processes (spatial thinking, prior awareness, motivation) in the initial visual information.

3. Formulation. Formulation of the answer's hypothesis to the research question (experience of using visual analytics, an ability to study and apply new language systems), formalization of new information.

Development of computer visualization technologies and their continuous complication create difficulties in interaction between a user and developed visualization tools [7]. This circumstance becomes critical in a situation where visualization tools provide cooperative participation of a group of researchers or are a way of exchanging information between specialists with different levels of training or area of specialization. There is a need to choose between users ability to use new visualization tools or involving existing visual communication skills to interpret data.

\section{Analytical visualization tools}

A significant part of tasks, where visualization tools are used, implicitly takes into account the assumption that the research question's answer can be obtained as a result of a single interpretation of visualized information by a researcher.
However, this is reasonable only for a number of problems, the formulation of which implies the possibility of setting a direct question and receiving an answer as a necessary result [6]. For many practical studies, analysis goal achievement is possible as a result of the sequential solution of a number of problems, including when formulation of the next intermediate problem is possible only after obtaining the solution to the previous problem [10].

An example of analysis tasks with similar properties is the interpretation of empirical data obtained during practical studies with a low level of formalization of the subject area [13]. In this case, the purpose of the ongoing visual analysis is to obtain new knowledge based on detection of internal patterns in the studied data and on the study of their properties. On this basis, similar tasks, which require consistent study, possibly with the use of various tools of analysis, are classified as analytical.

The planning task is considered as a study of the set of boundary requirements aimed at finding parameters of the set $O=\left\{O_{1} . . O_{n}\right\}$ that ensure the best results. The ambiguity of the target function becomes the reason for using analytical visualization tools that allow to a decision maker to clarify the problem statement while its solving. An additional circumstance, which involves the choice of interactive visualization tools of the problem, is the need to coordinate preliminary awareness of a potential user (previous experience) and visual research features.

\section{Visual representation function of the planning task}

Basing on the formulated requirements for visualization tools, which are necessary to solve the planning problem, a system for interactive presentation of data, which are included in the description of an arbitrary educational program, has been developed (Fig. 2). Three-dimensional visual model is proposed as a visualization tool designed to solve the problem, it forms a visual image of information objects included in the source data [11]. An informative object is an element of an educational program (an academic discipline, a course, program's section). Each such object is an array of data (name, course, duration, capacity, incoming requirements, planned results), including variables of different types.

There has been developed a software that allows to get an interpreted visual image with the use of visualizers of Autodesk $3 \mathrm{ds}$ Max package. The algorithm for constructing a visual image is implemented using Maxscript language, therefore it is portable and can be easily adapted to new visualization technical capabilities. Visualization interface only partially uses Autodesk 3ds Max environment and can be adapted to the needs of a particular user.

Reasonable interactive management system creates conditions for setting new research questions and receiving answers quickly, accelerating achievement of the analysis goal. Consequently, interactive features of the visualization tool determine sequence and logic of a researcher's reasoning.

To reduce the training period required to become familiar with the new interpretation tool, it is proposed to use a representation metaphor based on traditional methods of visualizing tabular data (charts, graphs). In 3D space of the visualization tool, a cylindrical coordinate system is defined, that allows each point in space to be matched with three values: training time, load, result. The scale of measurement units along time and result axes can be arbitrary, the load is measured as a percentage accordingly to the maximum possible. The ability to compare objects is realized through the use of color coding, which rules can be changed in accordance with perceptional characteristics of a particular user [12]. 


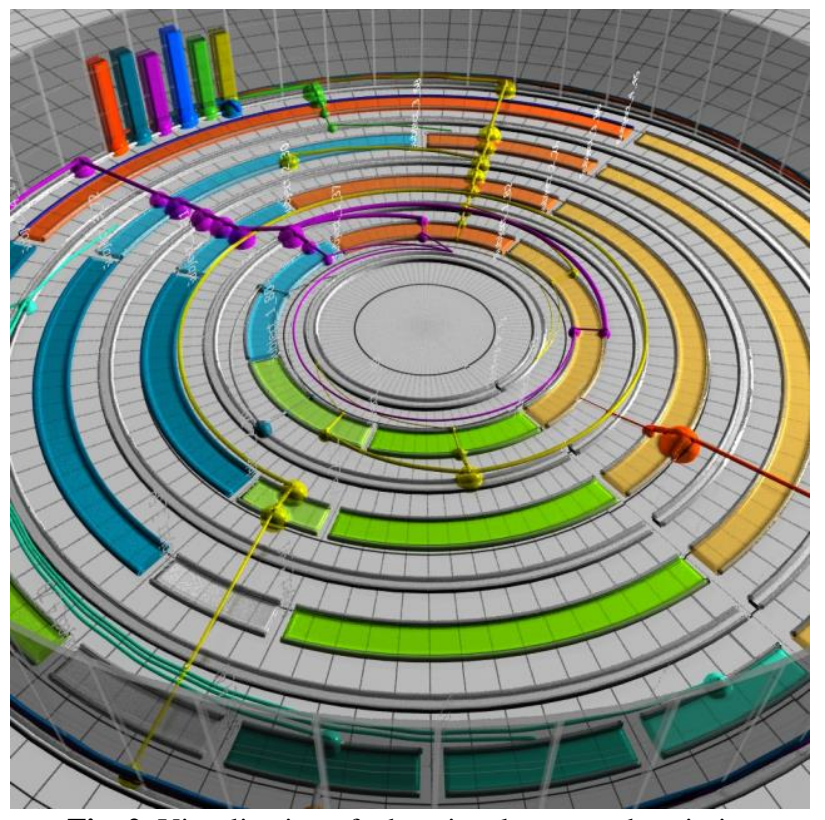

Fig. 2. Visualization of educational process description

The radial direction of the time axis allows to visualize educational programs data of any duration (bachelor's degree, specialist's program and master's degree). Program steps, which correspond to the given time intervals (years, semesters) are divided by concentric circular elements used to represent the accumulated results. Each concentric element is a reference scale $(0-100 \%)$ with a common starting point. The proposed structure provides a presentation of an increasing number of learning results without worsening general perception of data.

The information object provides to an observer an opportunity to interpret visual attributes as values of the corresponding parameters: color is an identification attribute, dimensions are load ones, position corresponds to the training period. Information about incoming requirements and planned learning results is presented as links between information objects. Attributes of such connections are their direction and quantity, which correspond to the source data. In accordance with characteristics of the subject area, incoming (requirements) and outgoing (results) communications have the same type, that means it can be interpreted as created or developing competencies.

For a visual presentation of accumulated learning results (Fig. 3), the simultaneous use of two expressive means is proposed: a rating scale of effectiveness and visual scaling of links. In the first case, it becomes possible to efficiently use the three-dimensional space of the visual model, in the second one the assessment of training results in the educational program can occur while interpreting two-dimensional visualization.

The method for visualizing intermediate and general results (Fig. 2) in the form of profiles for summing results was developed. The profile has the form of a histogram representing accumulated results as a percentage of planned values. To simplify the image of the studied data, elements of the results presentation can be temporarily excluded from the visual model.

Visual objects, which present to a user data of the achieved learning results, perform one of the main functions, which consists of searching for options of individual elements characteristics, which satisfy the condition (1). Thus, the use of visual presentation elements as a system of interactive control of the state of the visualization tool is proposed. The interface of interaction with the visualization tool created in this case becomes the basis for a cognitive interpretation of the source data images, taking into account individual characteristics of a user's thinking.

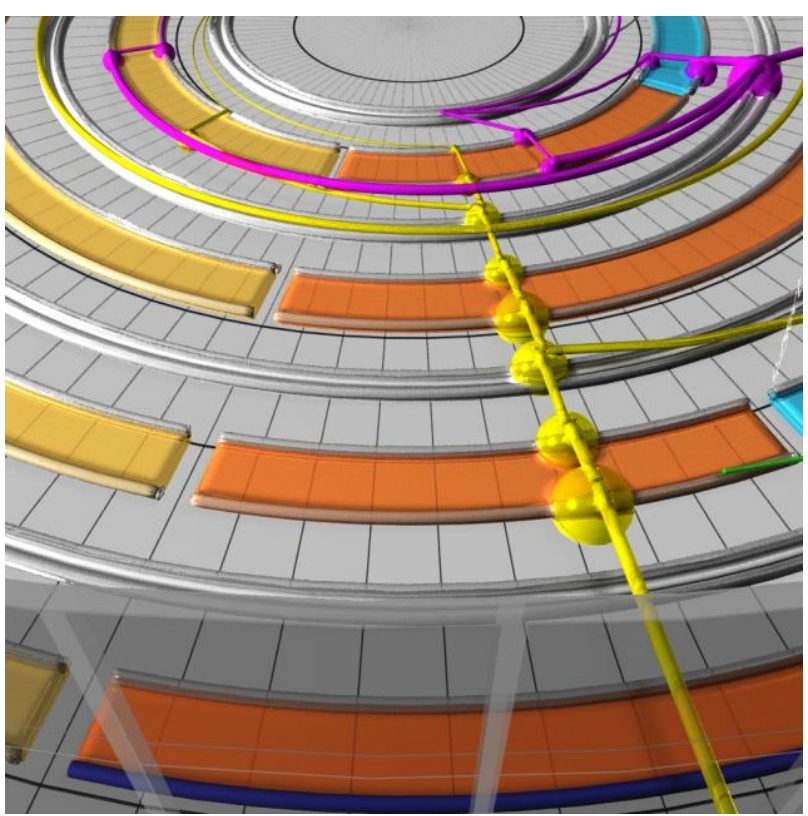

Fig. 3 Visualization of accumulated results

\section{Benefits of visualization tools}

Practical usage of the developed visualization tool made it possible to get an assessment of the proposed approach benefits comparing to traditional methods of interpreting and verifying heterogeneous data contained in documents, which regulate educational programs.

The proposed tool for visualizing an educational environment is supplemented by an ability to save options for solving planning problems and for corresponding real results. This creates conditions for a planning search for periods of varying lengths, during which the search and evaluation of factors that have necessary effect on the achieved results of the educational program are carried out.

Usage of visual analytics in this direction makes it possible to form a detailed idea about patterns of an educational environment functioning. The combination of visual design tools of an educational process and accumulation of knowledge about achieved results creates a unique information environment, access to which can be useful to both the "organizers" and the "participants" of this research process. Due to features of visualization tools, an interdisciplinary analytical approach for solving problems of designing complex multi-factor processes is formed. The potential benefits of this approach might be the following features:

- Reduction of time required to analyze large amount of heterogeneous data.

- Usage of highly specialized experience of specialists in the given subject area without the need of analysts participation who have skills in data analysis field.

- Possibility of organizing a collective solution to the planning problem, taking into account a significant amount of additional boundary conditions that are formed during the solution process.

- Formalization of knowledge related to the solution of the problem of planning the studied process, which provides effectiveness increase of solving similar problems in the future.

- Reduction of time required to a decision maker to complete the design process, based on the possibility of obtaining a complete picture of the studied data, as well as based on persuasiveness of visualization tools. 


\section{Usage prospects}

Perspective options for using user's interaction with the developed visualization tool can be considered as follows:

- Analysis of the set of profiles of intermediate results simultaneously with the general idea of the studied process, formed using the developed visualization tool, allows to make dynamic correction of the planned results.

- Identification and elimination of contradictions in the source data. Lack of input data: incoming requirements of the information object are not provided with results. The requested data are missing. It is detected as an open input of an information object (Fig. 4).

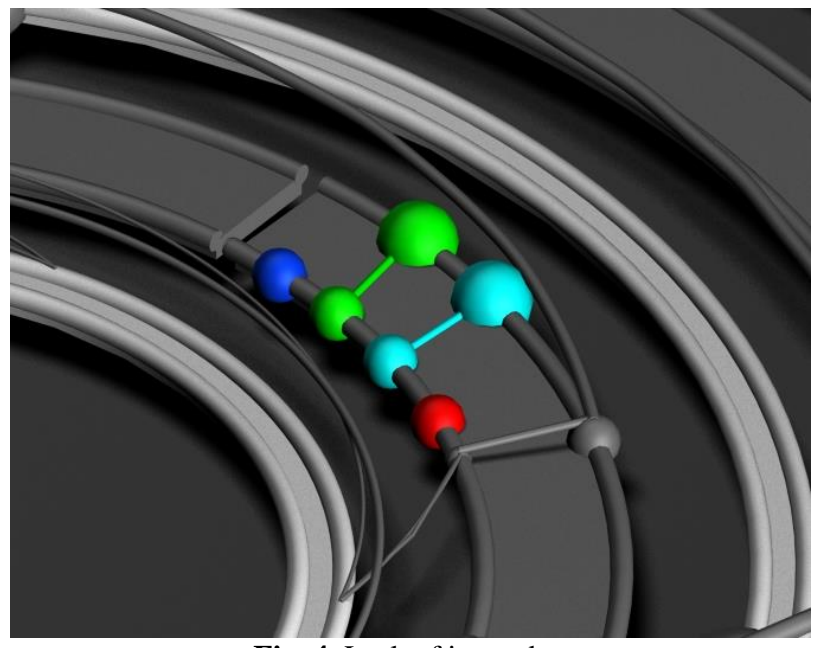

Fig. 4. Lack of input data

- Chronological discrepancy between incoming requirements of the information object and the results achieved. Contradiction arises if the input of the information object requires data that will be received later. It is visualized as a line of accumulated results going in the opposite direction (Fig. 5).

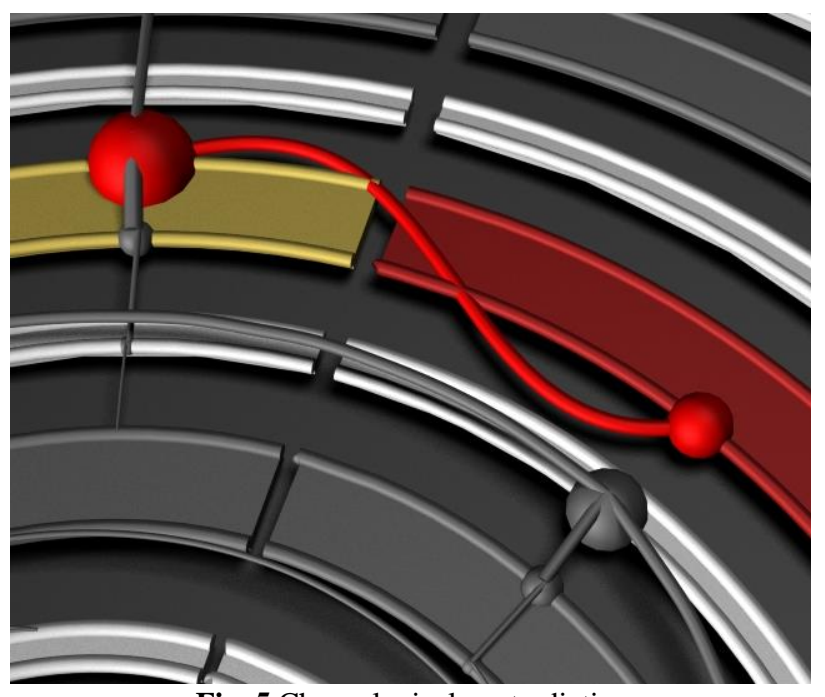

Fig. 5 Chronological contradiction

- Unreasonable usage of existing resources. The result of the interpretation of result lines that are not presented at the output of the overall process.

- Degradation of results. The proposed visualization tool takes into account possibility of reducing the level of the achieved result according to time. A contradiction is the results presence in the final profile that do not take into account the level of their degradation.
- Duplication of results. Resources usage for repeatedly obtaining similar results. The visual image of such a contradiction is diverging lines of cumulative results.

\section{Conclusion}

The use of visualization tools as a tool of operational data research in tasks planning is an example of visual analysis of heterogeneous data. The obtained advantages are high speed of visual perception, which is necessary for simultaneous comparison of large amount of disparate facts, as well as the ability to interpret data not only in numerical form.

- The proposed visualization tool allows to solve problems of an analytical type, to carry out sequential operations of obtaining, interpreting information, forming hypotheses, making decisions about changing goals or decision conditions.

- The technique of using visualization tools allows to achieve the research goal without interpreting numerical values. Thus, an approach to solving problems of this type, based solely on visualization tools, is proposed.

- A version of the source data visual presentation has been developed, which ensures a reduction of resource intensity of the user's training phase.

- The visualization tool has low dependence on the specifics of the task's subject area. Thus, experience of using the proposed means of interpretation can be involved in solving other problems.

- Low requirements for preliminary preparedness of users provide an ability to use visualization tools in case of absence of special training in solving problems of data interpretation, which have an interdisciplinary nature.

\section{Acknowledgements}

This work was supported by Russian Science Foundation, project 18-11-00215.

\section{References}

[1] Batch A., Elmqvist N. The Interactive Visualization Gap in Initial Exploratory Data Analysis. // IEEE Transactions on Visualization and Computer Graphics, 2018, Vol. 24, Issue 1, pp. 278-287. doi: 10.1109/TVCG.2017.2743990

[2] Blascheck T. [et al.]. VA2: A Visual Analytics Approach for Evaluating Visual Analytics Applications // IEEE Transactions on Visualization and Computer Graphics, 2016, Vol. 22, Issue 1, pp. 61-70. doi: 10.1109/TVCG.2015.2467871

[3] Cano S. [et al.]. A Visualization and Human-Computer Interaction Proposal in the Context of Pronunciation Information // IEEE Revista Iberoamericana de Tecnologias del Aprendizaje, 2016, Vol. 11, Issue 1, pp. 12-17. doi: 10.1109/RITA.2016.2518440

[4] Chen C. Top 10 Unsolved Information Visualization Problems // IEEE Computer Graphics and Applications, 2005, Vol. 25 Issue 4, pp. 12-16. doi: 10.1109/MCG.2005.91

[5] Chen M., Golan A. What May Visualization Processes Optimize? // IEEE Transactions on Visualization and Computer Graphics. 2016, Vol. 22, Issue 12, pp. 26192632. doi: 10.1109/TVCG.2015.2513410

[6] Crouser R.J. [et al.]. Toward Theoretical Techniques for Measuring the Use of Human Effort in Visual Analytic Systems // IEEE Transactions on Visualization and Computer Graphics, 2017, Vol. 23, Issue 1, pp. 121-130. doi: 10.1109/TVCG.2016.2598460

[7] Dasgupta A. [et al.]. Familiarity Vs Trust: A Comparative Study of Domain Scientists' Trust in Visual Analytics and 
Conventional Analysis Methods. // IEEE transactions on visualization and computer graphics, 2017, Vol. 23, Issue 1, pp. 271-280. doi: 10.1109/TVCG.2016.2598544

[8] Matsushita M., Kato T. Interactive Visualization Method for Exploratory Data Analysis // Proceedings Fifth International Conference on Information Visualisation, 2001. doi: 10.1109/IV.2001.942128

[9] Pirolli P.L. Information Foraging Theory: Adaptive Interaction with Information. Oxford University Press, 2007.

[10] Podvesovskii A.G., Isaev R.A. Visualization Metaphors for Fuzzy Cognitive Maps // Scientific Visualization, 2018, Vol. 10, No. 4, pp. 13-29. doi: 10.26583/sv.10.4.02

[11] Zakharova A.A. [et al.]. Visual modeling in an analysis of multidimensional data // IOP Conf. Series: Journal of Physics: Conf. Series, 2018, Vol. 944, 012127. doi: 10.1088/1742-6596/944/1/012127

[12] Zakharova A.A., Vekhter E.V., Shklyar A.V. The Applicability of Visualization Tools in the Meta-Design of an Educational Environment // European Journal of Contemporary Education, 2019. Vol. 8, No. 1, pp. 43-51. doi: 10.13187/ejced.2019.1.43

[13] Vieira C., Parsons P., Byrd V. Visual learning analytics of educational data: A systematic literature review and research agenda // Computers and Education, 2018, Vol. 122, pp. 119-135. doi: 10.1016/j.compedu.2018.03.018 